\title{
54. Synthetic Diet Increases Blood Protein in the Silkworm Bombyx mori*)
}

\author{
By Masaru Kato and Hiromi Yamada \\ Department of Zoology, Faculty of Science, Kyoto University \\ (Comm. by Eikiti Hiratsuka, M.J.A., March 13, 1967)
}

Since the studies of synthetic diet have been developed, it has always been in our minds that something might happen in the blood situation of the larvae fed on synthetic diet. Thus, it was found that the blood protein increases to the double amount of that in the larvae fed on mulberry leaves. This tendency was further investigated by changing the diet from the synthetic to mulberry leaves.

Experimental materials and methods. The $F_{1}$ of the cross of strains Gunko and Manri, kindly provided by Gunze Silk Co., was used in this experiment.

The composition of the synthetic diet used is given in the previous paper. ${ }^{1)}$ The feeding was carried out at a temperature of $25 \pm 2^{\circ} \mathrm{C}$ under aseptic condition. ${ }^{2)}$

Newly hatched larvae were divided into four groups $(A, B, C$, and $D$ ). Group-A was reared with fresh mulberry leaf alone. Both $B$ and $C$ groups were started on synthetic diet, but changed to mulberry leaf on the 1st day of the 3rd $(B)$ or of the 4 th $(C)$ instar. $D$ group was constantly reared with the synthetic diet throughout the investigation.

Ten milliliters of blood from each group was collected from 3-day old last instar larvae by cutting off the tip of the dorsal horn. Phenylthiourea was then added to the blood to prevent melanin formation. These blood samples were centrifuged to eliminate haemocytes before acid hydrolysis at $110^{\circ} \mathrm{C}$ for $22 \mathrm{hrs}$. The determinations of total and free amino acids were carried out with a Hitachi Model KLA-3 amino acid analyzer.

Results and discussion. The results obtained are shown in Table I and Fig. 1. While the amount of most amino acids hydrolized increases in the blood of $D$ group larvae fed exclusively on synthetic diet, there is no distinguishable increase in free amino acids as a whole. An examination of Table I suggests 3 patterns of increase for the total amino acids determined: 1) The amount of threonine, methionine, isoleucine, leucine, tyrosine, phenylalanine or arginine

*) No. 23 of the joint studies on artificial diet for the silkworm Bombyx mori by Hamamura et al. 


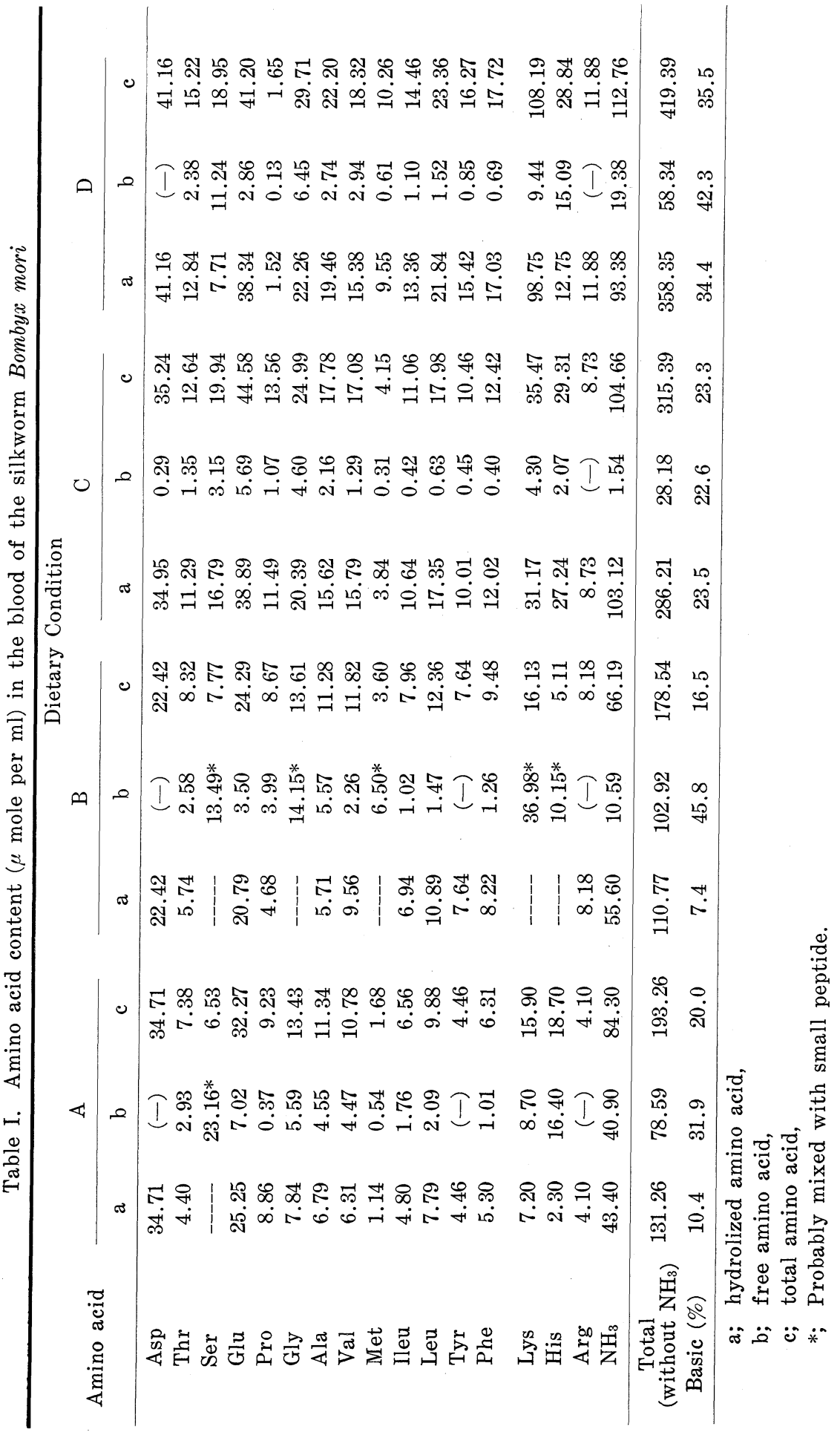


[Vol. 43,

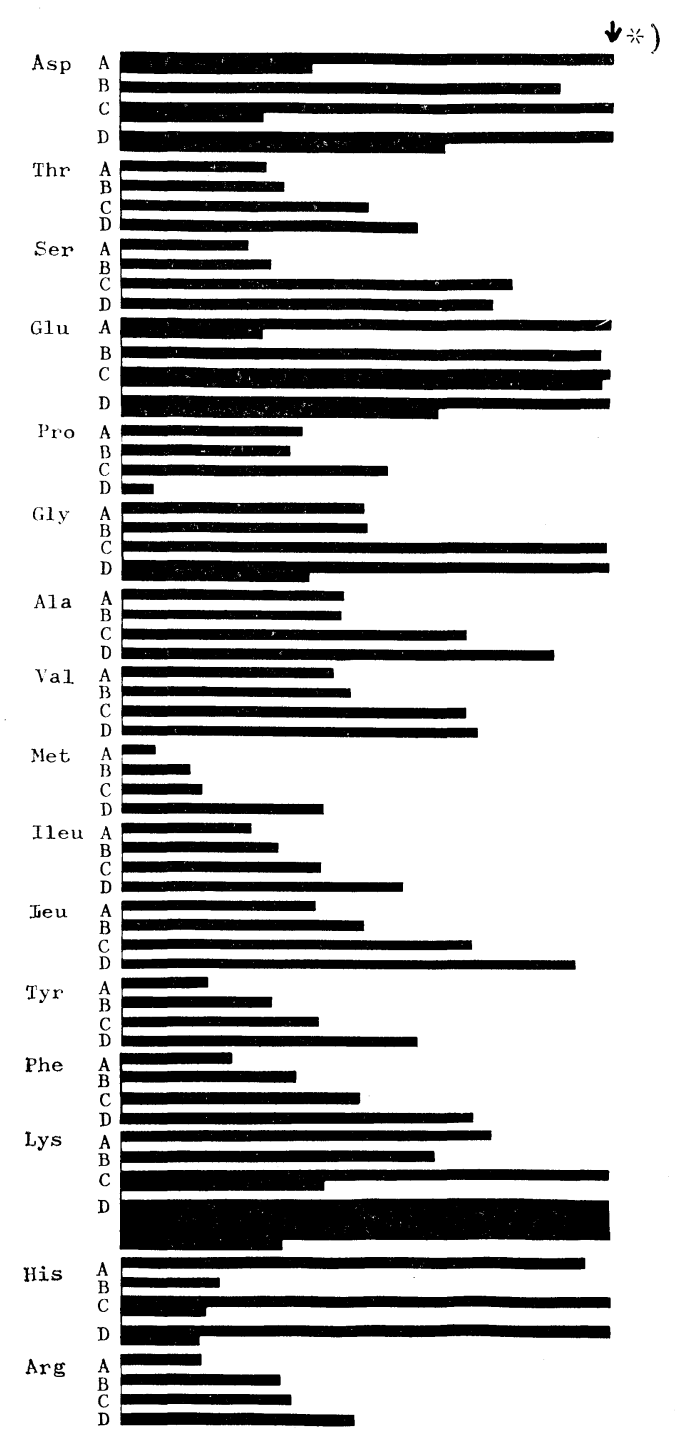

$\downarrow *) \quad 25 \mu$ mole per $\mathrm{ml}$,

A-D: Dietary condition.

Fig. 1. Content of total amino acid in the blood the silkworm Bombyx mori.

increases in the blood of $B$ group larvae changed from synthetic diet on the 1st day of the 3rd instar. 2) Serine, glutamic acid, glycine, alanine, valine and histidine increase in $C$ group larvae which were taken over by mulberry leaf on the 1st day of the 4th instar. 3) Lysine and aspartic acid increased particularly in $D$ group larvae fed on synthetic diet only (Table I and Fig. 1). However, a question arises as to the small amount of proline in the $D$ group. This 
might be due to some enzymatic reaction produced in this dietary condition..$^{3)}$ Using the present synthetic diet, in any case, the amino acid content of silkworm larva blood has, as a whole, been increased about twice over that of larvae fed on fresh leaf. This suggests that the present synthetic diet can be digested and absorbed to be readily converted to blood protein in this insect. This view is supported by the finding that the total nitrogen absorbed while feeding through the 5th instar was much more in larvae fed on synthetic diet than on fresh mulberry leaf:-175 $\mathrm{mg}$ and $84 \mathrm{mg}$ per individual on the average.

In a preliminary investigation on the amino acid content in the blood protein of this insect fed on an earlier prepared synthetic diet, ${ }^{4}$ it was found that every amino acid content was poor and the blood was much more watery than that of larvae fed on the present synthetic diet. On this earlier diet about $25 \%$ of larvae grew with an excessive 6 th instar, and only about half of newly hatched larvae could eventually produce very poor cocoons. It must be noted that the addition of chlorogenic acid and oleic acid instead of morin to the basal synthetic diet not only increases body weight and growth rate, but also absorption of amino acids through the intestine to construct blood proteins. It further seems likely that this tendency has already been determined in an early larval stage, at least before the 3rd instar (Table I and Fig. 1).

Of course, it is unknown how chlorogenic acid or oleic acid promotes the absorption of amino acid to construct the blood protein, but these findings suggest that chlorogenic acid or oleic acid is related to some special mechanism of amino acid metabolism in this insect.

Summary. Changing dietary conditions of the silkworm Bombyx mori from synthetic diet to mulberry leaf, the amount of most amino acids (particularly lysine) in the blood protein was remarkably increased. This tendency was found to be already determined before the 3rd instar for some amino acids. It is also suggested that the increase of blood protein may be due to some important role taken by chlorogenic acid or oleic acid added to the synthetic diet.

Acknowledgements. We wish to express our appreciation to Drs. K. Nakamura and Y. Hamamura for their valuable discussions and to our technical assistant Miss N. Sadamichi. Our thanks are also due to Drs. F. R. Harwood and T. Komai for reading this manuscript. 


\section{References}

1) Yamada, H., and Kato, M.: Proc. Japan Acad., 43, 230-233 (1967).

2) Kato, M., and Yamada, H.: Journal of Silkworm (France), 85, 15-16 (1963-1964).

3) Brosemer, R. W., and Veerabhadrappe, P. S.: Biochim. Biophys. Acta., 110, 102 (1965).

4) Hayashiya, K., Naito, K., Matsuura, K., Nishida, J., and Hamamura, Y.: J. Agr. Chem. Soc. Japan, 37, 160 (1963). 